\title{
Geometrically Nonlinear Free Axisymmetric Vibrations Analysis of Thin Circular Functionally Graded Plates Using Iterative and Explicit Analytical Solution
}

\author{
Rachid El Kaak and Khalid El Bikri \\ Mohammed $V$ University in Rabat, Ecole Normale Supérieure de l'Enseignement Technique de Rabat, \\ Département de génie mécanique, LaMIPI, B.P. 6207, Rabat Instituts, 10100 Rabat, Morocco
}

\begin{abstract}
Rhali Benamar
Mohammed V University in Rabat, Ecole Mohammadia d'Ingénieurs, LERSIM, Av. Ibn Sina, Rabat, Morocco.
\end{abstract}

(Received 14 December 2014; accepted 15 June 2015)

This paper deals with nonlinear free axisymmetric vibrations of functionally graded (FG) thin circular plates whose properties vary in thickness. The inhomogeneity of the plate is characterized by a power law variation of the Young's modulus and mass density of the material along the thickness direction, whereas Poisson's ratio is assumed to be constant. The theoretical model is based on Hamilton's principle and spectral analysis using a basis of admissible Bessel's functions to yield the frequencies of the circular plates under clamped boundary conditions on the basis of the classical plate theory. The large vibration amplitudes problem, reduced to a set of nonlinear algebraic equations, is solved numerically. The nonlinear to linear frequency ratios are presented for various values of the volume fraction index $n$ showing a hardening type nonlinearity. The distribution of the radial bending stresses associated to the nonlinear mode shape is also given for various vibration amplitudes and compared with those predicted by the linear theory. Then, explicit analytical solutions are presented, based on the semi-analytical model previously developed by El Kadiri et al. for beams and rectangular plates. This model allows direct and easy calculation for the first nonlinear axisymmetric mode shape with its associated nonlinear frequencies and nonlinear bending stresses of FG circular plates, which are expected to be very useful in engineering applications and in further analytical developments. An excellent agreement is found with the results obtained by the iterative method.

\section{INTRODUCTION}

The concept of functionally graded materials (FGMs) was first introduced in 1984 as ultrahigh-temperature resistant materials for aircraft, space vehicles, and other engineering applications. ${ }^{1}$ FGMs are nonconventional composite materials that are microscopically inhomogeneous, and their mechanical properties vary continuously in one or more directions. This is achieved by gradually varying the volume fraction of the constituent materials. The continuity of the material properties reduces the influence of the presence of abrupt interfaces and avoids high interfacial stresses. Furthermore, FGMs can be tailored to achieve particular desired properties, and the gradation in properties of materials can optimize the stress distribution.

Many studies have been devoted to FG plate vibrations in the literature. Allahverdizadeh et al. ${ }^{2}$ investigated the nonlinear free and forced vibration of thin circular FG plates. Praveen and Reddy ${ }^{3}$ conducted the nonlinear transient thermoelastic analysis of FG ceramic-metal plates using the finite element method. Yang and Shen ${ }^{4}$ examined the dynamic response of initially stressed FGM rectangular thin plates subjected to impulsive loads. The effects of the volume fraction index, the foundation stiffness, the plate aspect ratio, the shape and duration of the applied impulsive load on the dynamic response of FGM plates have been studied in this work. Also, the vibration characteristics and the transient response of shear-deformable
FGM plates made of temperature-dependent materials in thermal environments have been examined by Yang and Shen. ${ }^{5}$ The differential quadrature technique, the Galerkin approach, and the modal superposition method have been used to determine the transient response of the plate subjected to lateral dynamic loads. Huang and Shen ${ }^{6}$ discussed the nonlinear vibration and dynamic response of FG plates in a thermal environment by using an improved perturbation technique. The results reveal that the temperature field and the volume fraction distribution have significant effects on the nonlinear vibration and the dynamic response of simply supported rectangular plates with no in-plane displacements. Reddy and Cheng $^{7}$ studied the harmonic vibration problem of FG plates by means of a three-dimensional asymptotic theory formulated in terms of the transfer matrix. Prakash and Ganapathi ${ }^{8}$ analyzed the asymmetric flexural vibration and thermoelastic stability of FG circular plates. They used a finite element method to solve the problem. Efraim and Eisenberger ${ }^{9}$ studied the exact vibration analysis of thick annular isotropic and FGM plates of variable thicknesses. The motion equations that they obtained by the first order shear deformation theory have been solved by the exact element method. Dong ${ }^{10}$ presented an analysis of three-dimensional free vibration of FG annular plates via Chebyshev-Ritz method. Malekzadeh et al. ${ }^{11}$ discussed the in-plane free vibration of FG circular arches with 
temperature-dependent properties under a thermal environment. They assumed that the material properties and temperatures vary along the thickness direction, the governing equation and boundary conditions were obtained by the Hamilton principle. Viswanathan et al. ${ }^{12}$ studied free vibration of a symmetric angle-ply laminated annular circular plate of variable thickness using the first order shear deformation theory using spline function approximation. The equations of motion for the plates are derived using the first order shear deformation theory. The solutions of displacement functions are assumed in a separable form to obtain a system of coupled differential equations in terms displacement and rotational functions, and these functions are approximated by Bickley-type splines of order three. The vibration of 3- and 5-layered plates, made up of two types of materials and two types of boundary conditions, are considered. A generalized eigenvalue problem is obtained and solved numerically for an eigenfrequency parameter and for an associated eigenvector of spline coefficients. Ebrahimi ${ }^{13}$ studied geometrically nonlinear vibration of a piezoelectrically actuated FGM plate with an initial large deformation, based on Kirchhoff's-Love hypothesis with Von-Karman-type geometrical large nonlinear deformation. Recently, Kermani, Ghayour, and Mirdamad ${ }^{14}$ reported a free vibration analysis of multi-directional FG circular and annular plates using a semi analytical/numerical method called state space-based differential quadrature method. Viswanathan, Javed, and Aziz ${ }^{15}$ discussed the free vibration of laminated antisymmetric angle-ply annular circular plates with inclusion of the first order shear deformation theory using a spline function approximation by applying a point collocation method. The equations of motion of the plates are derived using first order shear deformation theory.

In the present work, the large axisymmetric free vibration amplitudes of clamped immovable thin FG circular plates is analyzed by using and adapting the model previously presented by Haterbouch and Benamar ${ }^{16,17}$ for large vibration amplitudes of isotropic circular plates. By assuming harmonic motion and expanding the transverse displacement in the form of the finite series of basic functions-namely the linear free vibration mode shape of the clamped circular plate-obtained in terms of Bessel's functions, the discretized expressions for the total strain energy and kinetic energy have been derived. In addition to the classical mass and rigidity tensors, a fourth order tensor appears due to the nonlinearity in these expressions. The application of Hamilton's principle reduced the large amplitude free vibration problem to a set of nonlinear algebraic equations, which have been solved numerically in each case, leading to the first nonlinear axisymmetric mode shape of clamped circular plates. The relationships between the nonlinear to linear frequency ratio have been obtained, as well as the mode shape and the non-dimensional maximum vibration amplitude for the first nonlinear mode shape of circular plates, showing hardening type nonlinearity and the dependence of the first mode shape on the amplitude of vibration.

\section{GENERAL FORMULATION}

\subsection{Problem Definition}

As mentioned above, FGMs are nonconventional composite materials whose mechanical properties vary continuously due to gradual change in the volume fraction of the constituent

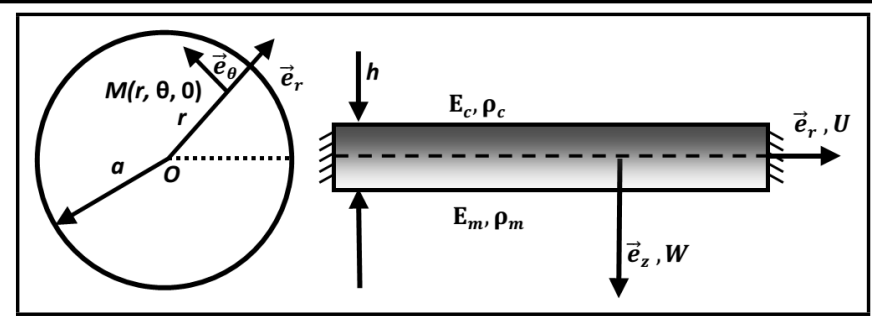

Figure 1. Geometry of FG clamped circular plate.

Table 1. Material properties used in the FG circular plate

\begin{tabular}{|c|c|c|}
\hline \multirow{2}{*}{ Material } & \multicolumn{2}{|c|}{ Property } \\
\cline { 2 - 3 } & $E(\mathrm{GPa})$ & $\rho\left(\mathrm{kg} / \mathrm{m}^{3}\right)$ \\
\hline Silicon nitride (Si3N4) & $355.2715 \mathrm{e} 9$ & 2370 \\
Stainless steel (SUS304) & $207.7877 \mathrm{e} 9$ & 8166 \\
\hline
\end{tabular}

material. In this study, a fully clamped thin circular plate of a uniform thickness $h$ and a radius $a$ is considered. The coordinate system is chosen so that the middle plane of the plate coincides with the polar coordinates $(r, \theta)$, the origin of the coordinate system being at the centre of the plate with the $z$-axis downward, the top surface of the plate is ceramic-rich, whereas the bottom surface is metal-rich, as depicted in Fig. 1.

\subsection{Mechanical Properties of FGCP}

FGMs are usually modelled as an inhomogeneous isotropic linear elastic material. However, here it is assumed that the material properties of FGM plates vary continuously through the plate thickness as a function of the volume fraction and the properties of constituent materials, from full ceramic at the top surface to full metal at the bottom. A power law distribution is used for the volume fraction of the constituents (metal and ceramic) as follows: ${ }^{18}$

$$
\begin{aligned}
& V_{m}(z)=\left(\frac{z}{h}+\frac{1}{2}\right)^{n} ; \\
& V_{c}(z)+V_{m}(z)=1 ;
\end{aligned}
$$

where subscripts $m$ and $c$ refer to the metal and ceramic constituents, respectively; $V_{m}$ and $V_{c}$ denote the volume fraction of metal and ceramic, respectively; $n$ is called the volume fraction index or material constant; and $z$ is the thickness coordinate $(-h / 2 \leq z \leq h / 2)$. Fully metal and fully ceramic are represented in Fig. 2, respectively, by zero and infinity values of the material constant $n$. Based on the linear role of the mixture, the effective mechanical properties of FGMs can be expressed as ${ }^{18}$

$$
P=P_{c} V_{c}+P_{m} V_{m}
$$

where $P_{m}$ and $P_{c}$ denote the specific properties of metallic and ceramic constituents, respectively. Therefore, all the mechanical and thermal properties of the FGM plate, such as Young's modulus, $E$, can be written as

$$
E(z)=E_{c}+\left(E_{m}-E_{c}\right) V_{m}
$$

Poisson's ratio, $\nu$, is assumed to be constant for simplicity and convenience.

In what follows, a metal, stainless steel (SUS304) and ceramics, silicon nitride (Si3N4) system of FGMs is considered. These material properties are given in Table 1 . 


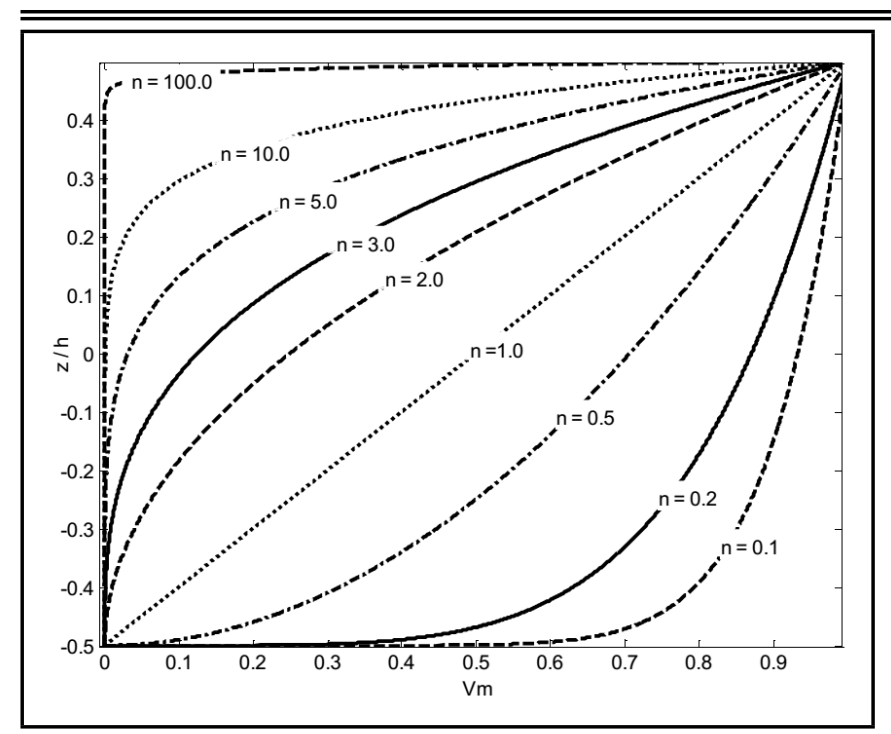

Figure 2. Volume fraction of metal along the thickness.

\subsection{Vibration Analysis}

Considering axisymmetric vibrations of the FG circular plate, the displacements are given in accordance with the classical plate theory:

$$
\begin{aligned}
& u_{r}(r, z, t)=U(r, t)-z \frac{\partial W_{(r, t)}}{\partial r}, \\
& u_{\theta}(r, t)=0, \quad u_{z}(r, t)=W(r, t) ;
\end{aligned}
$$

where $U$ and $W$ are the in-plane and out-of-plane displacements of the middle plane point $(r, \theta, 0)$ respectively, and $u_{r}$, $u_{\theta}$ and $u_{z}$ are the displacements along $\vec{e}_{r}, \vec{e}_{\theta}$ and $\vec{e}_{z}$ directions, respectively.

The non-vanishing components of the strain tensor in the case of large displacements are given by Von-Karman relationships:

$$
\{\varepsilon\}=\left\{\varepsilon^{0}\right\}+z\{K\}+\left\{\lambda^{0}\right\} ;
$$

in which $\left\{\varepsilon^{0}\right\},\{K\}$ and $\left\{\lambda^{0}\right\}$ are given by

$$
\begin{aligned}
& \left\{\varepsilon^{0}\right\}=\left[\begin{array}{c}
\varepsilon_{r}^{0} \\
\varepsilon_{\theta}^{0}
\end{array}\right]=\left[\begin{array}{c}
\frac{\partial U}{\partial r} \\
\frac{U}{r}
\end{array}\right] ; \\
& \{K\}=\left[\begin{array}{l}
K_{r} \\
K_{\theta}
\end{array}\right]=\left[\begin{array}{c}
-\frac{\partial^{2} W}{\partial r^{2}} \\
-\frac{1}{r} \frac{\partial W}{\partial r}
\end{array}\right] ; \\
& \left\{\lambda^{0}\right\}=\left[\begin{array}{c}
\lambda_{r} \\
\lambda_{\theta}
\end{array}\right]=\left[\begin{array}{c}
\frac{1}{2}\left(\frac{\partial W}{\partial r}\right)^{2} \\
0
\end{array}\right] ;
\end{aligned}
$$

For the FGM circular plate shown in Fig. 1, the stress can be expressed as:

$$
\{\sigma\}=[Q]\{\varepsilon\}
$$

in which $\{\sigma\}=\left[\sigma_{r} \sigma_{\theta}\right]^{T}$ and the terms of the matrix $[Q]$ can be obtained by the relationships given, for example, in Timoshenko, Weinsowsky-Krieger, and Jones. ${ }^{19}$ The force and moment resultants are defined by

$$
\begin{aligned}
& \left(N_{r}, N_{\theta}\right)=\int_{-h / 2}^{h / 2}\left(\sigma_{r}, \sigma_{\theta}\right) d z \\
& \left(M_{r}, M_{\theta}\right)=\int_{-h / 2}^{h / 2}\left(\sigma_{r}, \sigma_{\theta}\right) z d z
\end{aligned}
$$

The in-plane forces and bending moments in the plate are given by

$$
\left[\begin{array}{l}
N \\
M
\end{array}\right]=\left[\begin{array}{ll}
A & B \\
B & D
\end{array}\right]\left[\begin{array}{c}
\left\{\varepsilon^{0}\right\}+\left\{\lambda^{0}\right\} \\
\{K\}
\end{array}\right]
$$

$A, B$, and $D$ are the symmetric matrices given by the following equation:

$$
\left(A_{i j}, B_{i j}, D_{i j}\right)=\int_{-h / 2}^{h / 2} Q_{i j}\left(1, z, z^{2}\right) d z
$$

Here, the $Q_{i j}$ 's are the reduced stiffness coefficients of the plate. The expression for the bending strain energy $V_{b}$, the membrane strain energy $V_{m}$, the coupling strain energy $V_{c}$ and the kinetic energy $T$ are given by

$$
\begin{gathered}
V_{b}=\pi \int_{0}^{a} D_{11}\left[\left(\frac{\partial^{2} W}{\partial r^{2}}\right)^{2}+\frac{1}{r^{2}}\left(\frac{\partial W}{\partial r}\right)^{2}+\right. \\
\left.2 \frac{\nu}{r} \frac{\partial W}{\partial r} \frac{\partial^{2} W}{\partial r^{2}}\right] r d r \\
V_{m}=\pi \int_{0}^{a} A_{11}\left[\left(\frac{\partial U}{\partial r}\right)^{2}+\frac{\partial U}{\partial r}\left(\frac{\partial W}{\partial r}\right)^{2}+\frac{1}{4}\left(\frac{\partial W}{\partial r}\right)^{4}+\right. \\
\left.\frac{U^{2}}{r^{2}}+\frac{2 \nu U}{r} \frac{\partial U}{\partial r}+\nu \frac{U}{r}\left(\frac{\partial W}{\partial r}\right)^{2}\right] r d r ; \\
V_{c}=\pi \int_{0}^{a}-B_{11}\left[\frac{\partial^{2} W}{\partial r^{2}}\left(\frac{\partial W}{\partial r}\right)^{2}+\frac{\nu}{r} \frac{\partial W}{\partial r}\left(\frac{\partial W}{\partial r}\right)^{2}\right] r d r
\end{gathered}
$$

and

$$
T=\pi I_{0} \int_{0}^{a}\left(\frac{\partial W}{\partial r}\right)^{2} r d r
$$

where $I_{0}$ is the inertial term given by

$$
I_{0}=\int_{-h / 2}^{h / 2} \rho(z) d z
$$

An approximation has been adopted in the present work consisting of neglecting the contribution of the in-plane displacement $U$ in the membrane strain energy expression. Such an assumption has been made when calculating the first two nonlinear mode shapes of circular plates and fully clamped rectangular plates. ${ }^{16,20,21,23}$ For the first nonlinear mode shape, the range of validity of this assumption has been discussed in the light of the experimental and numerical results obtained for the nonlinear frequency-amplitude dependence and the nonlinear bending stress estimates obtained at large vibration amplitude. $^{20,22}$ In order to examine the effects of large vibration amplitudes on the membrane stress patterns for clamped circular plates, the contribution of the in-plane displacement $U$ should be taken into account in the membrane strain expression. The assumption introduced above leads to

$$
V_{m}=\frac{\pi A_{11}}{4} \int_{0}^{a}\left(\frac{\partial W}{\partial r}\right)^{4} r d r
$$


The total strain energy, $V$, is then given by

$$
\begin{aligned}
V=\pi \int_{0}^{a} \frac{A_{11}}{4}\left(\frac{\partial W}{\partial r}\right)^{4}-B_{11}\left[\frac{\partial^{2} W}{\partial r^{2}}\left(\frac{\partial W}{\partial r}\right)^{2}+\right. \\
\left.\frac{\nu}{r} \frac{\partial W}{\partial r}\left(\frac{\partial W}{\partial r}\right)^{2}\right]+D_{11}\left[\left(\frac{\partial^{2} W}{\partial r^{2}}\right)^{2}+\right. \\
\left.\frac{1}{r^{2}}\left(\frac{\partial W}{\partial r}\right)^{2}+2 \frac{\nu}{r} \frac{\partial W}{\partial r} \frac{\partial^{2} W}{\partial r^{2}}\right] r d r
\end{aligned}
$$

\subsubsection{Discretization of the Total Strain and Kinetic Energy Expressions}

If the space and time functions are supposed to be separable and harmonic motion is assumed, the transverse displacement $W$ can be written as

$$
W(r, t)=w(r) \sin (\omega t)
$$

The spatial function $w(r)$ is expanded in the form of a finite series of $n$ basic functions $w_{i}(r)$ as follows:

$$
w(r)=a_{i} w_{i}(r)
$$

in which the usual summation convention for the repeated index $i$ is used over the range $[1, n]$. The transverse displacement $W(r, t)$ is then given by:

$$
W(r, t)=a_{i} w_{i}(r) \sin (\omega t) .
$$

The discretization of the total strain and kinetic energy expressions is made by substituting the expression for $W(r, t)$ given in Eq. (24) into Eqs. (18)-(21) and rearranging. This leads to the following expressions:

$$
\begin{aligned}
V= & \frac{1}{2} a_{i} a_{j} k_{i j} \sin ^{2}(\omega t)+\frac{1}{2} a_{i} a_{j} a_{k} c_{i j k} \sin ^{3}(\omega t)+ \\
& \frac{1}{2} a_{i} a_{j} a_{k} a_{l} b_{i j k l} \sin ^{4}(\omega t) ; \\
T= & \frac{1}{2} \omega^{2} a_{i} a_{j} m_{i j} \cos ^{2}(\omega t) ;
\end{aligned}
$$

in which $m_{i j}, k_{i j}, b_{i j k l}$ and $c_{i j k}$ are the mass tensor, the linear rigidity tensor, the fourth order nonlinear rigidity tensor and the third order nonlinear coupling tensor, respectively. The expressions for these tensors are

$$
\begin{aligned}
& m_{i j}=2 \pi I_{0} \int_{0}^{a} w_{i} w_{j} r d r \\
& k_{i j}=2 \pi D_{11} \int_{0}^{a}\left(\frac{d^{2} w_{i}}{d r^{2}} \frac{d^{2} w_{j}}{d r^{2}}+\frac{1}{r^{2}} \frac{d w_{i}}{d r} \frac{d w_{j}}{d r}+\right. \\
& \left.2 \frac{\nu}{r} \frac{d w_{i}}{d r} \frac{d^{2} w_{j}}{d r^{2}}\right) r d r \\
& c_{i j k}=-2 \pi B_{11} \int_{0}^{a}\left(\frac{d^{2} w_{i}}{d r^{2}} \frac{d w_{j}}{d r} \frac{d w_{k}}{d r}+\frac{\nu}{r} \frac{d w_{i}}{d r} \frac{d w_{j}}{d r} \frac{d w_{k}}{d r}\right) r d r \\
& b_{i j k l}=\frac{\pi A_{11}}{2} \int_{0}^{a}\left(\frac{d w_{i}}{d r} \frac{d w_{j}}{d r} \frac{d w_{k}}{d r} \frac{d w_{l}}{d r}\right) r d r
\end{aligned}
$$

\subsubsection{Formulations of the Governing Equations}

The dynamic behavior of the structure is governed by Hamilton's principle, which is symbolically written as

$$
\delta \int_{0}^{\pi / 2 \omega}(V-T) d t=\delta \emptyset=0 .
$$

Replacing $T$ and $V$ by their discretized expressions given by Eqs. (25) and (26) in the energy condition Eq. (28), integrating the time functions in the range $\left[0, \frac{\pi}{2 \omega}\right]$ and calculating the derivatives with respect to the $a_{i}$ 's leads to the following equation:

$$
\begin{gathered}
\frac{3 \pi}{32 \omega} a_{j} a_{k} a_{l} b_{r j k l}+\frac{3 \pi}{32 \omega} a_{i} a_{k} a_{l} b_{i r k l}+\frac{3 \pi}{32 \omega} a_{i} a_{j} a_{l} b_{i j r l}+ \\
\frac{3 \pi}{32 \omega} a_{i} a_{j} a_{k} b_{i j k r}-\left(\frac{\pi}{8 \omega} a_{j} m_{r j} \omega^{2}+\frac{\pi}{8 \omega} a_{i} m_{i r} \omega^{2}\right)+ \\
\left(\frac{\pi}{8} a_{j} k_{r j}+\frac{\pi}{8} a_{i} k_{i r}\right)+\left(\frac{2}{6 \omega} a_{j} a_{k} c_{r j k}+\frac{2}{6 \omega} a_{i} a_{k} c_{i r k}+\right. \\
\left.\frac{2}{6 \omega} a_{i} a_{j} c_{i j r}\right)=0 .
\end{gathered}
$$

The precedent equation can be rewritten as:

$$
\begin{gathered}
\left(\frac{\pi}{4 \omega} a_{i} k_{i r}\right)+\left(\frac{3 \pi}{8 \omega} a_{i} a_{j} a_{k} b_{i j k r}\right)+\left(\frac{1}{\omega} a_{i} a_{j} c_{i j r}^{s}\right)- \\
\left(\frac{\pi}{4 \omega} a_{i} m_{i r} \omega^{2}\right)=0
\end{gathered}
$$

It appears from Eqs. (27a)-(27b) and (27d) that the tensors $m_{i j}$ and $k_{i j}$ are symmetric, and that the fourth order tensor $b_{i j k l}$ is such that

$$
b_{i j k l}=b_{k l i j}=b_{j i l k}=b_{i k j l} .
$$

On the other hand, a third order tensor $c_{i j k}^{s}$ is defined by Eq. (29) such that

$$
c_{i j k}^{s}=\frac{1}{3}\left(c_{k i j}+c_{i k j}+c_{j i k}\right) .
$$

Consequently, Eq. (28) reduces to the following set of nonlinear algebraic equations:

$$
a_{i} k_{i r}+\frac{3}{2} a_{i} a_{j} a_{k} b_{i j k r}+\frac{4}{\pi} a_{i} a_{j} c_{i j r}^{s}-\omega^{2} a_{i} m_{i r}=0
$$

for $r=1, \ldots, n$. This can be written in a matrix form as

$$
\begin{gathered}
\{A\}^{T}[K]\{A\}+\frac{3}{2}\{A\}^{T}[B(A)]\{A\}+\frac{4}{\pi}\{A\}^{T}[C(A)]\{A\}- \\
\omega^{2}\{A\}^{T}[M]\{A\}=0 .
\end{gathered}
$$

Pre-multiplying Eq. (30) by the vector $(A)^{T}=\left[\begin{array}{lll}a_{1} & a_{2} \ldots a_{n}\end{array}\right]$ leads to the following expression for $\omega^{2}$ :

$$
\omega^{2}=\frac{a_{i} a_{j} k_{i j}+\frac{3}{2} a_{i} a_{j} a_{k} a_{l} b_{i j k l}+\frac{4}{\pi} a_{i} a_{j} a_{k} c_{i j k}^{s}}{a_{i} a_{j} m_{i j}} .
$$

To simplify the analysis and the numerical treatment of the set of nonlinear algebraic equations, a non-dimensional formulation has been considered by putting the spatial displacement function as

$$
w_{i}(r)=h w_{i}^{*}\left(r^{*}\right) ;
$$


where $r^{*}=r / a$, the non-dimensional radial coordinate, and Eq. (30) may be rewritten in non-dimensional form as

$$
2 a_{i} k_{i r}^{*}+3 a_{i} a_{j} a_{k} b_{i j k r}^{*}+\frac{8}{\pi} a_{i} a_{j} c_{i j r}^{s *}-2 \omega^{* 2} a_{i} m_{i r}^{*}=0 .
$$

The $m_{i j}^{*}, k_{i j}^{*}, c_{i j k}^{*}$ and $b_{i j k l}^{*}$ terms are non-dimensional tensors related to the dimensional ones by the following relationships:

$$
\begin{aligned}
& m_{i j}=2 \pi I_{0} h^{2} a^{2} m_{i j}^{*} ; \\
& k_{i j}=\frac{2 \pi D_{11} h^{2}}{a^{2}} k_{i j}^{*} ; \\
& c_{i j k}=\frac{-2 \pi B_{11} h^{3}}{a^{2}} c_{i j k}^{*} ; \\
& b_{i j k l}=\frac{\pi A_{11} h^{4}}{2 a^{2}} b_{i j k l}^{*} .
\end{aligned}
$$

These non-dimensional tensors are defined by

$$
\begin{aligned}
& m_{i j}^{*}= \int_{0}^{a} w_{i}^{*} w_{j}^{*} r^{*} d r^{*} \\
& k_{i j}^{*}= \int_{0}^{a}\left(\frac{d^{2} w_{i}^{*}}{d r^{* 2}} \frac{d^{2} w_{j}^{*}}{d r^{* 2}}+\frac{1}{r^{* 2}} \frac{d w_{i}^{*}}{d r^{*}} \frac{d w_{j}^{*}}{d r^{*}}+\right. \\
&\left.2 \frac{\nu}{r^{*}} \frac{d w_{i}^{*}}{d r^{*}} \frac{d^{2} w_{j}^{*}}{d r^{* 2}}\right) r^{*} d r^{*} ; \\
& c_{i j k}^{*}=\beta \int_{0}^{a}\left(\frac{d^{2} w_{i}^{*}}{d r^{* 2}} \frac{d w_{j}^{*}}{d r^{*}} \frac{d w_{k}^{*}}{d r^{*}}+\frac{\nu}{r^{*}} \frac{d w_{i}^{*}}{d r^{*}} \frac{d w_{j}^{*}}{d r^{*}} \frac{d w_{k}^{*}}{d r^{*}}\right) r^{*} d r^{*} ; \\
& b_{i j k l}^{*}=\alpha \int_{0}^{a}\left(\frac{d w_{i}^{*}}{d r^{*}} \frac{d w_{j}^{*}}{d r^{*}} \frac{d w_{k}^{*}}{d r^{*}} \frac{d w_{l}^{*}}{d r^{*}}\right) r^{*} d r^{*} ;
\end{aligned}
$$

where $\omega^{*}$ is the non-dimensional nonlinear frequency parameter defined by

$$
\omega^{* 2}=\gamma \omega^{2}
$$

in which $\omega^{* 2}$ is given by the following expression:

$$
\omega^{* 2}=\frac{a_{i} a_{j} k_{i r}^{*}+\frac{3}{2} a_{i} a_{j} a_{k} a_{l} b_{i j k r}^{*}+\frac{4}{\pi} a_{i} a_{j} a_{k} c_{i j k}^{s *}}{\omega^{* 2} a_{i} a_{j} m_{i r}^{*}} .
$$

The parameters $\alpha, \beta$, and $\gamma$ are given by

$$
\begin{aligned}
& \alpha=\frac{A_{11} h^{2}}{4 D_{11}} \\
& \beta=\frac{-B_{11} h}{D_{11}} \\
& \gamma=\frac{I_{0} a^{4}}{D_{11}}
\end{aligned}
$$

\subsection{Bending Stress Expressions}

The bending strains $\varepsilon_{b r}$ and $\varepsilon_{b \theta}$ are given by

$$
\begin{aligned}
& \varepsilon_{b r}(z)=-z\left(\frac{d^{2} w}{d r^{2}}\right) \\
& \varepsilon_{b \theta}(z)=-z\left(\frac{1}{r} \frac{d w}{d r}\right) .
\end{aligned}
$$

By using the classical thin plate assumption of plane stress and Hooke's law, the radial and circumferential bending
Table 2. Non-dimensional linear frequencies, associated with the axisymmetric modes of a clamped FG circular plate for $i=1, \ldots, 6$.

\begin{tabular}{|c|c|c|c|c|c|c|}
\hline$i$ & 1 & 2 & 3 & 4 & 5 & 6 \\
\hline$\left(\omega_{l}^{*}\right)_{i}$ & 10.21 & 39.77 & 89.10 & 158.18 & 247.00 & 355.56 \\
\hline
\end{tabular}

stresses are given by

$$
\begin{aligned}
& \sigma_{b r}=-\frac{z E(z)}{\left(1-\nu^{2}\right)}\left[\left(\frac{d^{2} w}{d r^{2}}\right)+\nu\left(\frac{1}{r} \frac{d w}{d r}\right)\right] ; \\
& \sigma_{b \theta}=-\frac{z E(z)}{\left(1-\nu^{2}\right)}\left[\left(\frac{1}{r} \frac{d w}{d r}\right)+\nu\left(\frac{d^{2} w}{d r^{2}}\right)\right] .
\end{aligned}
$$

In terms of the non-dimensional parameters defined in the previous section, the radial and circumferential bending stresses $\sigma_{b r}$ and $\sigma_{b \theta}$ can be defined by

$$
\begin{aligned}
& \sigma_{b r}=-\frac{z^{*} E\left(z^{*}\right) h^{2}}{\left(1-\nu^{2}\right) a^{2}}\left[\left(\frac{d^{2} w^{*}}{d r^{* 2}}\right)+\nu\left(\frac{1}{r^{*}} \frac{d w^{*}}{d r^{*}}\right)\right] ; \\
& \sigma_{b \theta}=-\frac{z^{*} E\left(z^{*}\right) h^{2}}{\left(1-\nu^{2}\right) a^{2}}\left[\left(\frac{1}{r^{*}} \frac{d w^{*}}{d r^{*}}\right)+\nu\left(\frac{d^{2} w^{*}}{d r^{* 2}}\right)\right] .
\end{aligned}
$$

\section{NUMERICAL RESULTS AND DISCUSSION}

\subsection{Numerical Details}

The basic functions $w_{i}^{*}$ to be used in the expansion series of w in Eq. (23) must satisfy the theoretical clamped boundary conditions (i.e., zero displacement and zero slopes along the circular edge). Since the linear problem of free axisymmetric flexural vibration of a clamped circular plate has an exact analytical solution, the chosen basic functions $w_{i}^{*}$ were taken as the linear free oscillation mode shapes of fully clamped circular plates given by ${ }^{24}$

$$
w_{i}^{*}\left(r^{*}\right)=A_{i}\left[J_{0}\left(\beta_{i} r^{*}\right)-\frac{J_{0}\left(\beta_{i}\right)}{I_{0}\left(\beta_{i}\right)} I_{0}\left(\beta_{i} r^{*}\right)\right] .
$$

where $\beta_{i}$ is the $i^{\text {th }}$ real positive root of the transcendental equation

$$
J_{1}(\beta) I_{0}(\beta)+J_{0}(\beta) I_{1}(\beta)=0 ;
$$

in which $J_{n}$ and $I_{n}$ are, respectively, the Bessel and the modified Bessel functions of the first kind and of order $n$. The parameter $\beta_{i}$ is related to the $i^{\text {th }}$ non-dimensional linear frequency parameter $\left(\omega_{l}^{*}\right)_{i}$ of the plate by

$$
\beta_{i}^{2}=\left(\omega_{l}^{*}\right)_{i}
$$

Leissa's study ${ }^{25}$ features examples of numerical values of $\left(\omega_{l}^{*}\right)_{i}$, and the first six values are given here in Table 2. $A_{i}$ is chosen such that

$$
\int_{0}^{1} w_{i}^{* 2} r^{*} d r^{*}=1
$$

Therefore, a set of orthonormal functions, and the mass tensor associated with the chosen transverse displacement is given by

$$
m_{i j}^{*}=\int_{0}^{1} w_{i}^{*} w_{j}^{*} r^{*} d r^{*}=\delta_{i j}
$$

where $\delta_{i j}$ is the Kronecker delta symbol. The first six basic functions $w_{i}^{*}, i=1, \ldots, 6$ are plotted in Fig. 3 . 


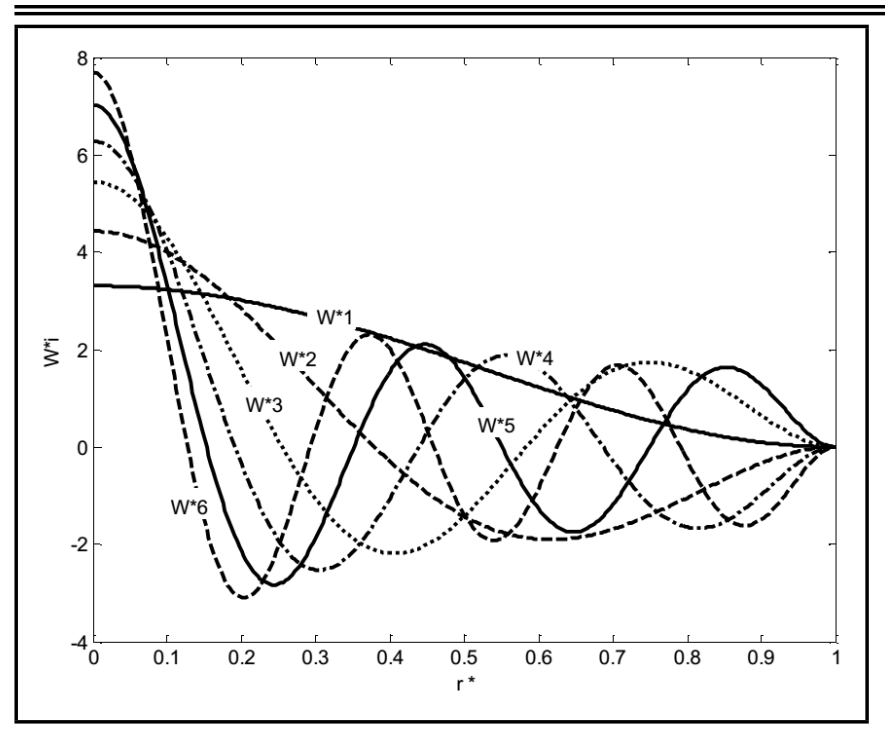

Figure 3. Axisymmetric out-of plane natural modes of vibration for a clamped circular plate $W_{i}^{*}$ for $i=1, \ldots, 6$.

\subsection{Iterative Method of Solutions and Anal- ysis of Numerical Results}

\subsubsection{Iterative Method of Solutions}

The set of nonlinear algebraic Eqs. (37) has been solved numerically by using the Harwell library routine NS01A, based on a hybrid iterative method combining the steepest descent and Newton's methods to obtain the numerical results presented for the first nonlinear mode shape of a thin FG circular plate. This method does not require a very good initial estimate of the solution or a step procedure, similar to other methods described for beams and rectangular plates. ${ }^{20-23}$ It was adopted here to ensure rapid convergence when varying the amplitude, which allowed solutions to be obtained with a quite reasonable number of iterations. The fundamental nonlinear mode shape was calculated in the neighborhood of the linear solution corresponding to a small numerical value of the coefficient $a_{r 0}\left(r_{0}=1\right)$ of the basic function $w_{r 0}^{*}$. The resulting solution was then used as an initial estimate for the following step corresponding to higher values of $a_{r 0}\left(r_{0}=1\right)$.

\subsubsection{Numerical Results and Discussion}

The first six linear axisymmetric eigenfunctions of the model presented above were used, respectively, to obtain the first nonlinear axisymmetric mode shape. The results obtained numerically from iterative solutions of the nonlinear algebraic system Eq. (37) are summarized in Table 3. The computed values of $a_{2}, a_{3}, \ldots, a_{6}$ obtained for assigned values of $a_{1}$ varying from 0.005 to 0.75 correspond to maximum dimensionless vibration amplitudes varying from 0.0165 to 2.386 and are given in Table 3. In each table, $a_{i}$ represents the contribution of the $i^{\text {th }}$ basic function $w_{i}^{*}$. The variable $w_{\max }^{*}$ is the maximum non-dimensional amplitude, and $\left(\omega_{n l}^{*} / \omega_{l}^{*}\right)$ is the ratio of the non-dimensional nonlinear frequency parameter defined in Eq. (41) to the corresponding non-dimensional linear frequency parameter given in Table 3. It can be seen from this table that the non-dimensional nonlinear frequencies calculated here from the nonlinear analysis for low amplitudes of up to 0.04 (very small values of $a_{1}$ and $a_{2}$ ) coincide exactly with the corresponding linear ones. Also, near to the linear frequency of a given mode, only the corresponding basic function has
Table 4. Effect of large vibration amplitudes on the frequencies of the first non-linear axisymmetric mode shape of a clamped FG circular plate $(n=$ $0.5)$.

\begin{tabular}{|c|c|c|c|}
\hline \multicolumn{4}{|c|}{ Non-linear frequency ratio } \\
\hline$W_{\max }^{*}$ & $n$ & Present work & From graph $^{2}$ \\
\hline 0.2 & & 1.0076 & 1.0074 \\
0.4 & & 1.0300 & 1.0259 \\
1.0 & 0.5 & 1.1752 & 1.1629 \\
1.5 & & 1.3626 & 1.3370 \\
\hline
\end{tabular}

Table 5. Frequency ratio $\left(\omega_{n l}^{*} / \omega_{l}^{*}\right)$ of a clamped isotropic circular plate $(n=$ $0.0)$.

\begin{tabular}{|c|c|c|c|c|c|}
\hline$W_{\max }^{*}$ & $2008^{2}$ & $2003^{16}$ & $1961^{26}$ & $1962^{27}$ & Present analysis \\
\hline 0.2 & 1.0075 & 1.0072 & 1.0070 & 1.0079 & 1.0108 \\
0.4 & 1.0296 & 1.0284 & 1.0278 & 1.0313 & 1.0421 \\
0.8 & 1.1135 & 1.1073 & 1.1065 & 1.1194 & 1.1560 \\
1.0 & 1.1724 & 1.1615 & 1.1617 & 1.1808 & 1.2318 \\
1.5 & 1.3567 & 1.3255 & 1.3343 & 1.3711 & 1.4542 \\
\hline
\end{tabular}

a significant contribution. At large vibration amplitudes, the mode contributions and the resonance frequency increase with the amplitude of vibration. The corresponding rate of increase decreases with the order of the mode considered and becomes negligible for the higher modes.

\subsubsection{Amplitude Frequency Dependence}

The dependence of the nonlinear frequency on the nondimensional vibration amplitude is listed in Table 4 for the first nonlinear axisymmetric mode shape of the FG circular plate for $n=0.5$. From this table, it is observed that the nonlinear frequency increases with increasing vibration amplitudes. It can be also observed that the results calculated via the present model exhibit a higher increase of the frequency compared with those obtained by Haterbouch and Benamar ${ }^{2}$ with a discrepancy of $6.22 \%$ for a value of the non-dimensional amplitude $w_{\max }^{*}=1.0$ and $0.41 \%$ for a non-dimensional vibration amplitude $w_{\max }^{*}=0.2$. This may be attributed to the negligence of in-plane displacements in the present theory. Table 5 shows the comparison of the nonlinear frequency ratio of the first mode shape of the isotropic circular plates (case of $n=0$ ) with those obtained for some isotropic circular plates in the literature.

Figure 4 shows the dependence of the frequency ratio of the clamped FG circular plate on the amplitude of vibration for various values of the power law index $n$. As may be seen in this figure, by increasing the values of the power law index in the range $[0,2]$, the frequency increases. For values higher than $n=2.0$, the frequency decreases when $n$ increases. This may be expected, since when the power law index $n=0.0$ or $n=1000.0$, the material is pure metallic or pure ceramic, respectively, and the non-dimensional frequency corresponds to the isotropic material case.

\subsubsection{Amplitude Dependence of the First Nonlin- ear Axisymmetric Mode Shape of FG Circular Plates}

Previous studies ${ }^{20-23}$ have shown that the nonlinear mode shapes of beam- and plate-like structures are amplitude dependent. This effect is illustrated in the present case in Fig. 5 in which the normalized nonlinear mode shapes of the first axisymmetric mode of the clamped FG circular plate are plotted for various values of the maximum non-dimensional amplitudes. All curves show the amplitude dependence of the first 
Table 3. Contribution coefficients to the first non-linear axisymmetric mode shape of the clamped FG circular plate, obtained numerically from iterative solution of the non-linear system in Eq. (37).

\begin{tabular}{||c|c|c|c|c|c|c|c||}
\hline \hline$w_{\max }^{*}$ & $\omega_{n l}^{*} / \omega_{l}^{*}$ & $a_{1}$ & $a_{2}$ & $a_{3}$ & $a_{4}$ & $a_{5}$ & $a_{6}$ \\
\hline 0.0165 & 1.0000 & 0.005 & -5.0666 E-008 & 9.3961 E-009 & -2.5725 E-009 & 8.0453 E-011 & -3.8763 E-013 \\
0.0330 & 1.0003 & 0.01 & -3.2327 E-007 & 1.0681 E-007 & -3.7776 E-009 & 8.9118 E-010 & -3.1623 E-010 \\
0.0661 & 1.0012 & 0.02 & -2.6017 E-006 & 8.6160 E-007 & -2.5008 E-008 & 3.1887 E-009 & -5.8694 E-010 \\
0.1322 & 1.0048 & 0.04 & -1.0800 E-008 & 7.3254 E-009 & -5.5800 E-009 & 5.9800 E-009 & -1.0800 E-010 \\
0.2641 & 1.0190 & 0.08 & -1.6245 E-004 & 5.4556 E-005 & -1.8763 E-006 & 2.8340 E-007 & -4.5036 E-008 \\
0.3299 & 1.0294 & 0.10 & -3.1274 E-004 & 1.0588 E-004 & -3.9480 E-006 & 6.4564 E-007 & -9.7508 E-008 \\
0.4934 & 1.0643 & 0.15 & -1.0059 E-003 & 3.4978 E-004 & -1.6334 E-005 & 3.2094 E-006 & -4.5234 E-007 \\
0.8165 & 1.1646 & 0.25 & -4.0663 E-003 & 1.5171 E-003 & -1.0883 E-004 & 2.7996 E-005 & -4.2409 E-006 \\
1.1347 & 1.2924 & 0.35 & -9.4441 E-003 & 3.8097 E-003 & -3.8014 E-004 & 1.1767 E-004 & -2.1055 E-005 \\
1.4495 & 1.4366 & 0.45 & -1.6788 E-002 & 7.2922 E-003 & -9.2628 E-004 & 3.2968 E-004 & -6.9137 E-005 \\
1.7625 & 1.5906 & 0.55 & -2.5619 E-002 & 1.1875 E-002 & -1.8036 E-003 & 7.1673 E-004 & -1.7152 E-004 \\
2.0746 & 1.7507 & 0.65 & -3.5523 E-002 & 1.7397 E-002 & -3.0261 E-003 & 1.3140 E-003 & -3.5021 E-004 \\
2.3862 & 1.9151 & 0.75 & -4.6185 E-002 & 2.3678 E-002 & -4.5770 E-003 & 2.1363 E-003 & -6.2198 E-004 \\
\hline
\end{tabular}

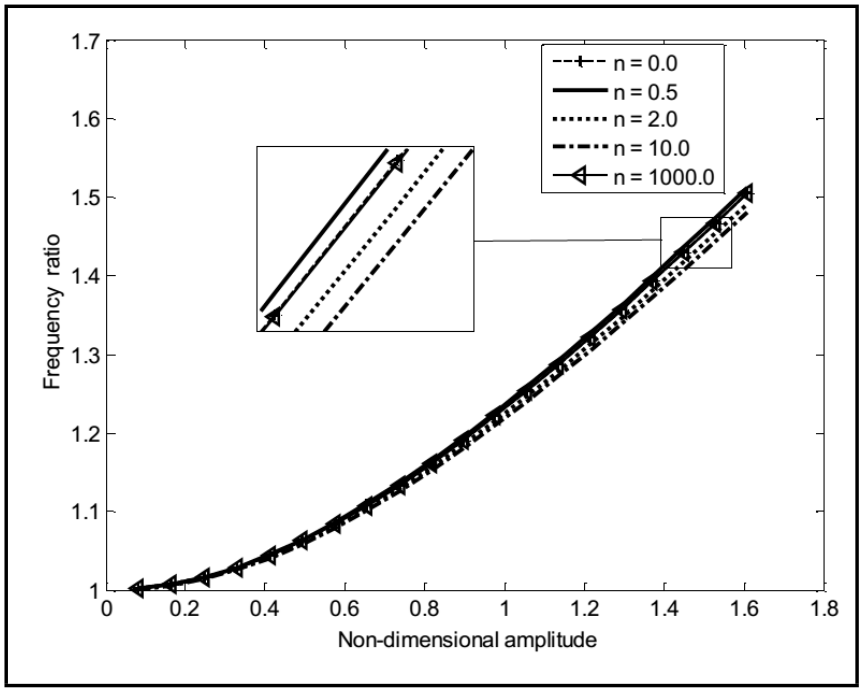

Figure 4. Effect of the power law index $n$ on the variation of the non-linear frequency ratios $\left(\omega_{n l}^{*} / \omega_{l}^{*}\right)$ of the clamped FG circular plate with the amplitude of vibration.

axisymmetric nonlinear mode shape with an increase of curvatures near to the clamped edges, which may lead one to expect that the bending stress near to the edges of the plate increases nonlinearly with the increase of the vibration amplitude. This is examined in the next subsection.

\subsubsection{Analysis of the Bending Stress Distribution As- sociated with the First Nonlinear Axisymmetric Mode Shape of FG Circular Plates}

As mentioned above, the present multimodal model allows not only determination of the amplitude-frequency dependence, but also the deformation of the mode shape due to the geometrical nonlinearity. From this last result, it was expected that the effect of the amplitude of vibration on the distribution of the associated bending stress would be of a great significance since the bending stress is related to the derivatives of the amplitude dependent transverse mode shape.

The radial bending stress distributions associated with the first axisymmetric nonlinear mode shape with nondimensional radius are plotted in Fig. 6. It can also be seen in Fig. 7 that the nonlinear radial bending stresses exhibit a higher increase near to the clamped edge compared with that expected in linear theory. The rate of increase in the radial bending stress is about 1.52, the rate of increase expected in linear theory for the first mode.

Figures 8-9 present the effect of the volume fraction index on the bending stress at the clamped edge and the plate center

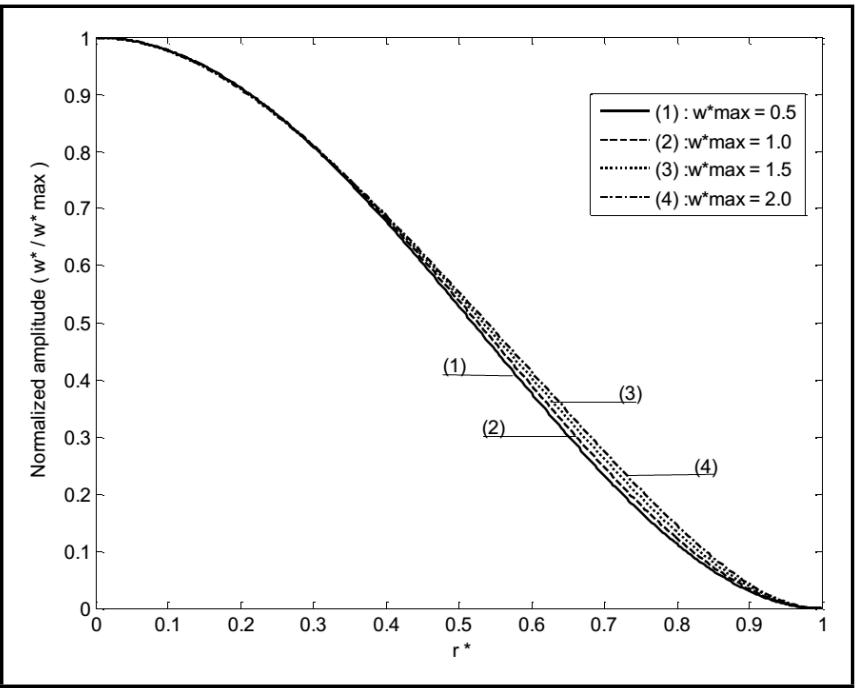

Figure 5. Normalized first non-linear axisymmetric mode shape of FG circular plate at various non-dimensional amplitudes and the power index $n=0.5$.

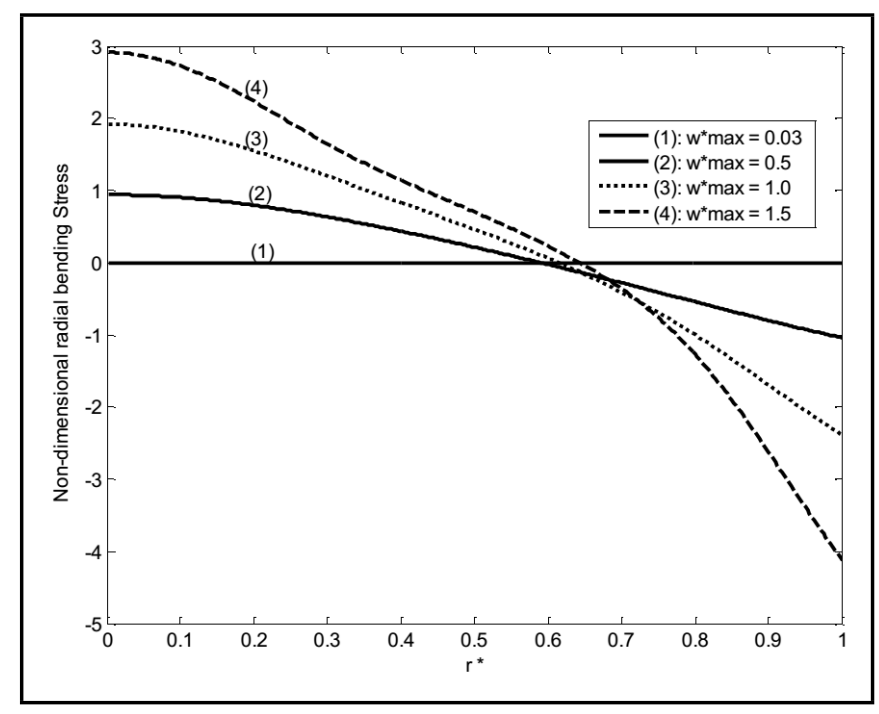

Figure 6. Non-dimensional radial bending stress associated to the first nonlinear axisymmetric mode shape of a clamped FG circular plate for $n=0.5$ and various non-dimensional vibration amplitudes.

through the plate thickness. It is obvious that by increasing the gradient index $(n)$, the variation of Young's modulus becomes increasingly abrupt through the thickness and, consequently, the stress varies accordingly. It is observed that the stress variation through the plate thickness is linear for the completely ceramic-rich and metal-rich plates corresponding, respectively, to $n=0.0$ and $n=\infty$, while the behavior is nonlinear and 


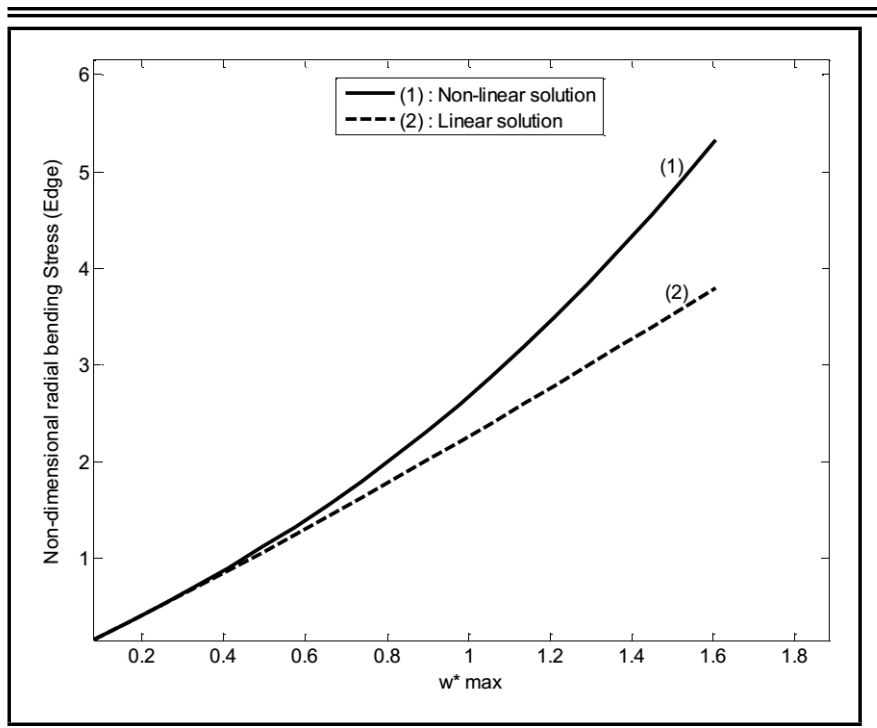

Figure 7. Effects of large vibration amplitudes on the non-dimensional radial bending stress at the edge of the FG circular plate.

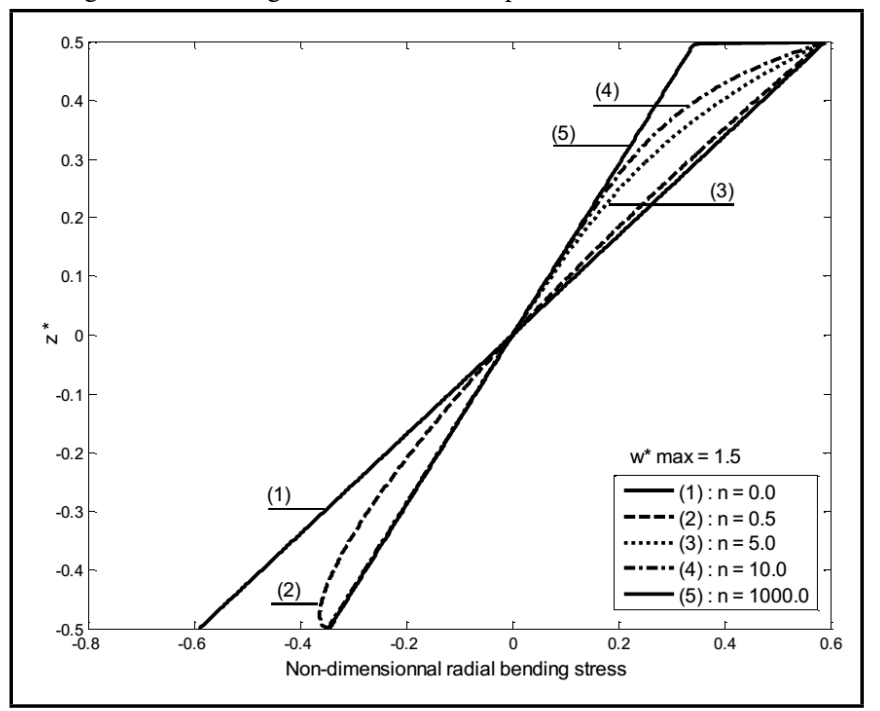

Figure 8. Effect of the volume fraction index $n$ on the radial bending stress through the thickness at the clamped center of the FG circular plate.

is governed by the variation of the properties in the thickness direction for the FGCP. Figure 10 depicts the variation related to the bending stress of a clamped FG circular plate at different levels of the plate cross-section for the power law index $n=0.5$ and the vibration amplitude $W_{\max }^{*}=1.5$.

\subsubsection{Explicit Analytical Solution}

The purpose here is to replace the iterative method of the solution of the set of the nonlinear algebraic equation, Eq. (37), necessary to obtain the clamped FG circular plate nonlinear axisymmetric mode shape and associated nonlinear resonant frequencies at large vibration amplitudes by an explicit solution, which may be appropriate for engineering purposes or for further analytical investigations. This explicit solution is obtained by applying and adapting the so-called first formulation developed for many beams and plates cases studied by El Kadiri, Benamar, and White ${ }^{28}$ and El Kadiri and Benamar. ${ }^{29}$ A comparison is then made between the two solutions-numerical iterative and analytical - in order to determine exactly the range of validity of the last approximate approach. To illustrate the method, the fundamental nonlinear mode shape is considered

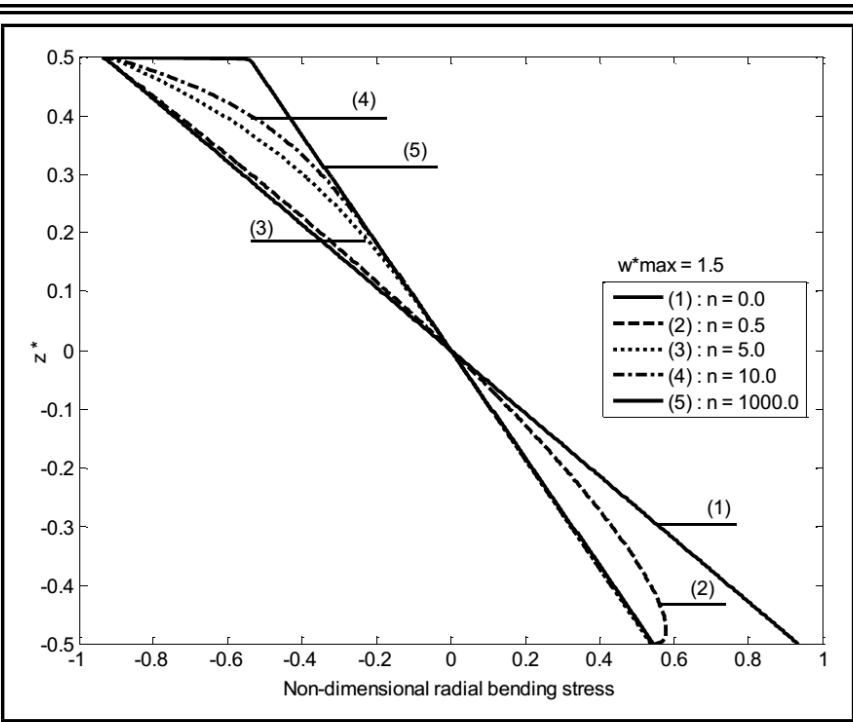

Figure 9. Effect of the volume fraction index $n$ on the radial bending stress through the thickness at the clamped Edge of the FG circular plate.

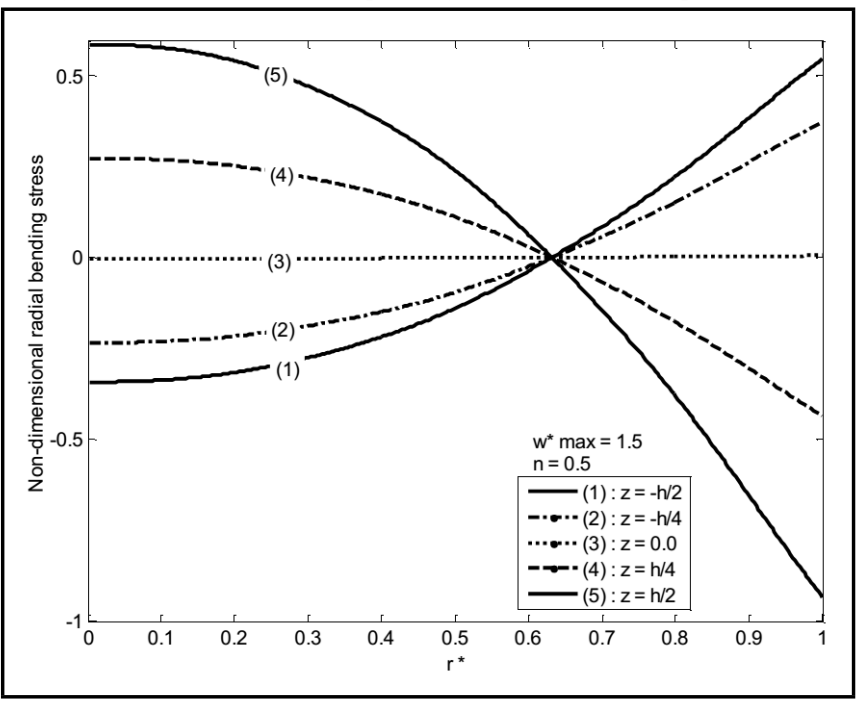

Figure 10. Variation of the radial bending stress at different levels of FG circular plate for $n=0.5$.

Table 6. Numerical values of the modal parameters $k_{i i}^{*}$ and $b_{111 i}^{*}$.

\begin{tabular}{|c|c|c|}
\hline$i$ & $k_{i i}^{*}$ & $b_{111 i}^{*}$ \\
\hline 1 & 104.363105549862 & 421.176182024113 \\
2 & 1581.74423079117 & 320.895319824783 \\
3 & 7939.54845205673 & -562.906703667013 \\
4 & 25022.2457661498 & 51.9601847057999 \\
5 & 61012.1806626721 & -15.6321082304476 \\
6 & 126429.530992020 & 6.09018059687781 \\
\hline
\end{tabular}

here by taking $r_{0}=1$. The analysis for the higher nonlinear modes would proceed similarly. A less constraining assumption, compared to the single mode approach, is made by neglecting in the expression $a_{i} a_{j} a_{k} b_{i j k r}^{*}$, appearing in Eq. (37), which leads to a simple formulation, leading to explicit expression for the amplitude dependence first nonlinear mode of the FG circular plates.

\subsubsection{Explicit Expression for the Amplitude Depen- dence First Nonlinear Mode of FG Circular Plate}

The first formulation is based on an approximation, which consists in neglecting in the expression $a_{i} a_{j} a_{k} b_{i j k r}^{*}$ of Eq. (37) when the first FG circular plate nonlinear mode is examined, 


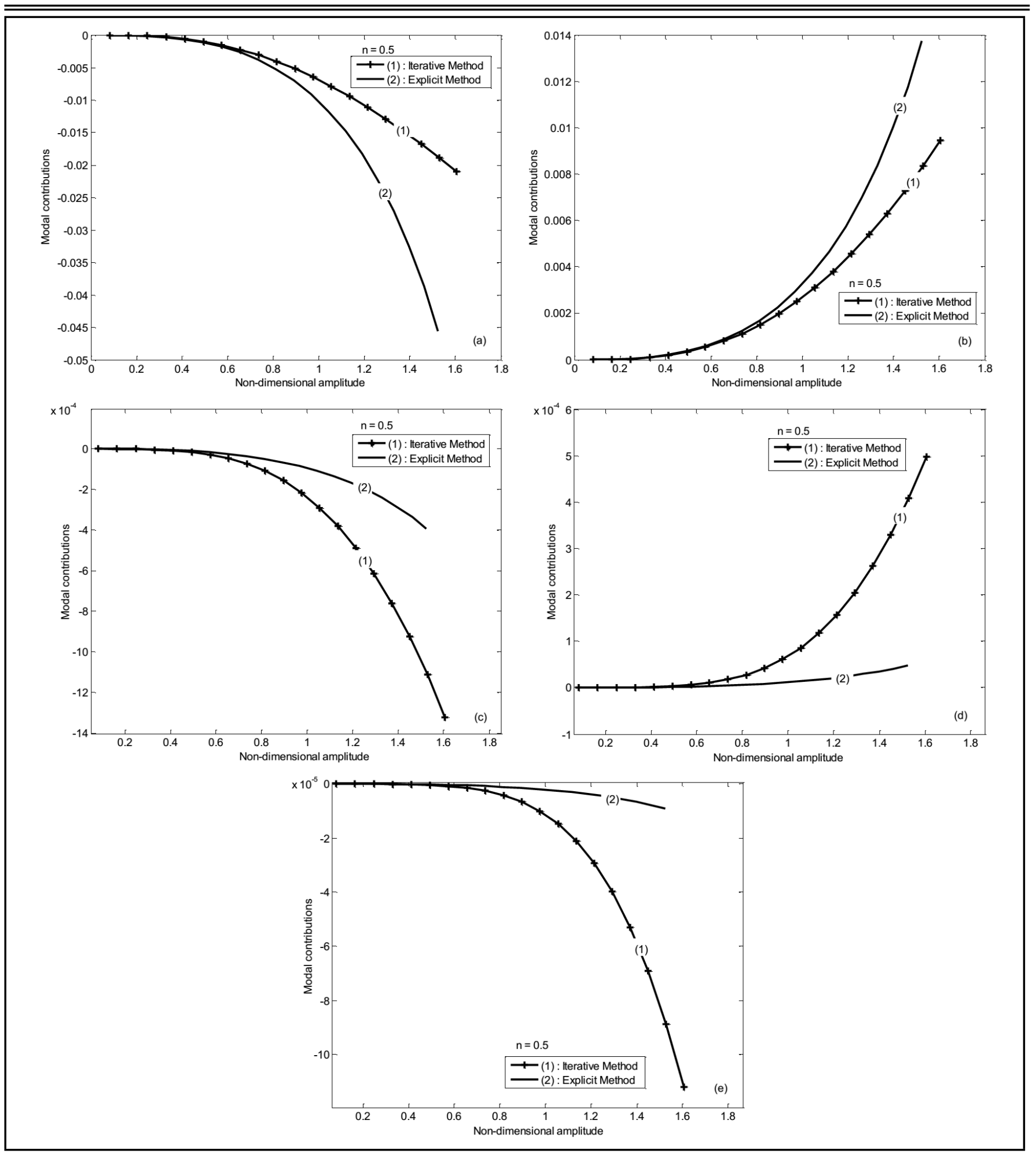

Figure 11. Comparison between the values of the modal contributions of the first non-linear mode shape of the FG circular plate.

the first, second and third order terms with respect to $\varepsilon_{i}$, i.e. the terms of the type $a_{1}^{2} \varepsilon_{k} b_{11 k r}^{*}, a_{1} \varepsilon_{j} \varepsilon_{k} b_{1 j k r}^{*}$, or $\varepsilon_{i} \varepsilon_{j} \varepsilon_{k} b_{i j k r}^{*}$, so that the only remaining term is $a_{1}^{3} b_{111 r}^{*}$. Thus, Eq. (37) becomes

$$
\left(k_{i r}^{*}-\omega_{n l 1}^{* 2} m_{i r}^{*}\right) \varepsilon_{i}+\frac{3}{2} a_{1}^{3} b_{111 r}^{*}=0
$$

for $r=2, \ldots, 6$, in which the repeated index $i$ is summed over the range $[1,6]$. Since the use of linear FG circular plate mode shapes as basic functions leads to diagonal mass and rigidity matrices, the above system permits one to obtain explicitly the basic function contributions $\varepsilon_{2}, \ldots, \varepsilon_{6}$ of the second and higher functions corresponding to a given value of the assigned first basic function contribution $a_{1}$, as follows:

$$
\varepsilon_{r}=-\frac{3}{2} \frac{a_{1}^{3} b_{111 r}^{*}}{\left(k_{r r}^{*}-\omega_{n l 1}^{* 2} m_{r r}^{*}\right)}
$$

where $r=2, \ldots, 6$.

The $\varepsilon_{r}$ 's, $(r \neq 1)$, depend on the known parameter $m_{r r}^{*}$, $k_{r r}^{*}, b_{111 r}^{*}$; the assigned value $a_{1}$, and the nonlinear frequency parameter $\omega_{n l 1}^{*}$. To express simply $\omega_{n l 1}^{* 2}$ with an acceptable accuracy, the single-function formula obtained from Eq. (41), 


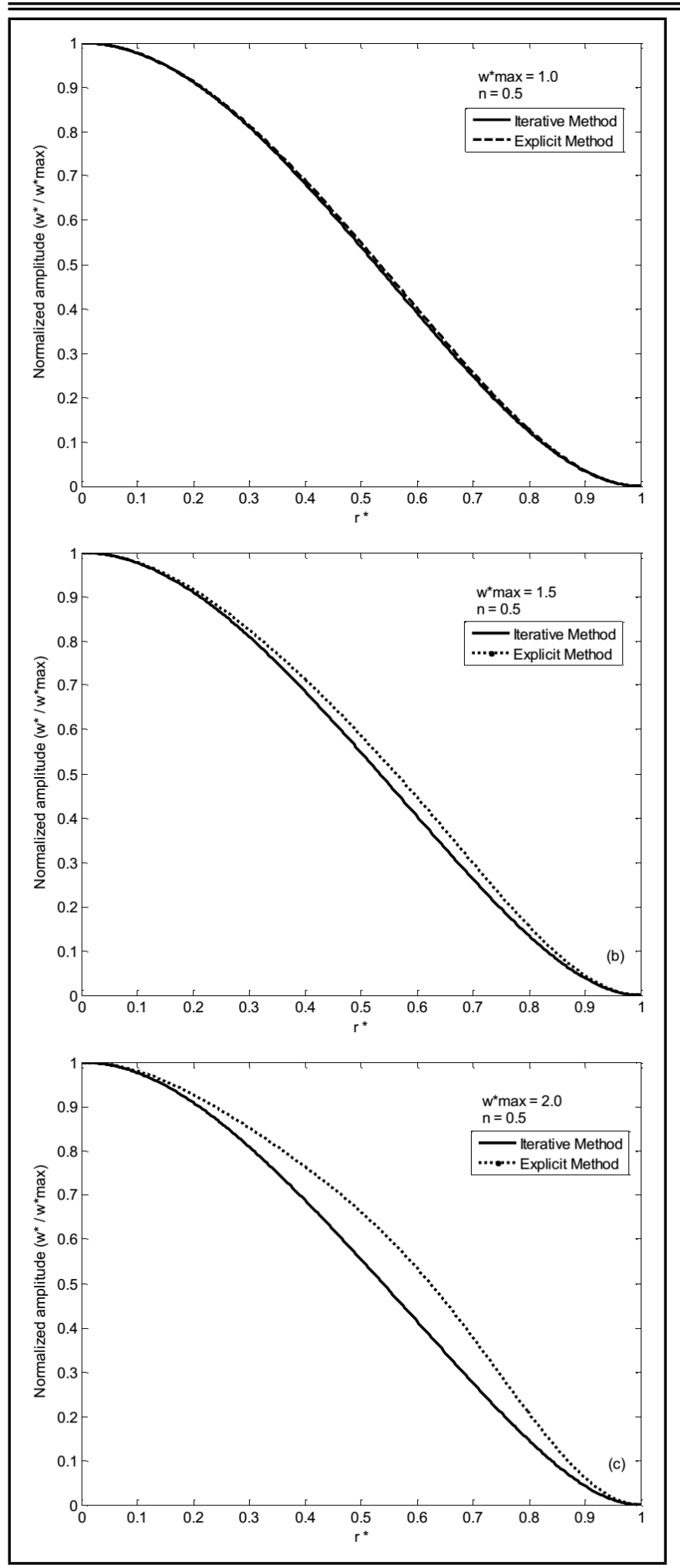

Figure 12. Comparison between the normalized radial section of the first nonlinear axisymmetric mode shape of a clamped FG circular plate for the power law index $n=0.5$ and various non-dimensional amplitudes. (-): Iterative method of solution. (- - -): Explicit analytical solution.

in which all of the $a_{i}$ 's, except $a_{1}$, are taken equal to zero, and is used as follows:

$$
\omega_{n l 1}^{* 2}=\frac{k_{11}^{*}}{m_{11}^{*}}+\frac{3 b_{1111}^{*}}{2 m_{11}^{*}} a_{1}^{2} .
$$

In the case considered here, the mass matrix is identical to the identity matrix $m_{11}^{*}=m_{r r}^{*}=1$, and by substituting

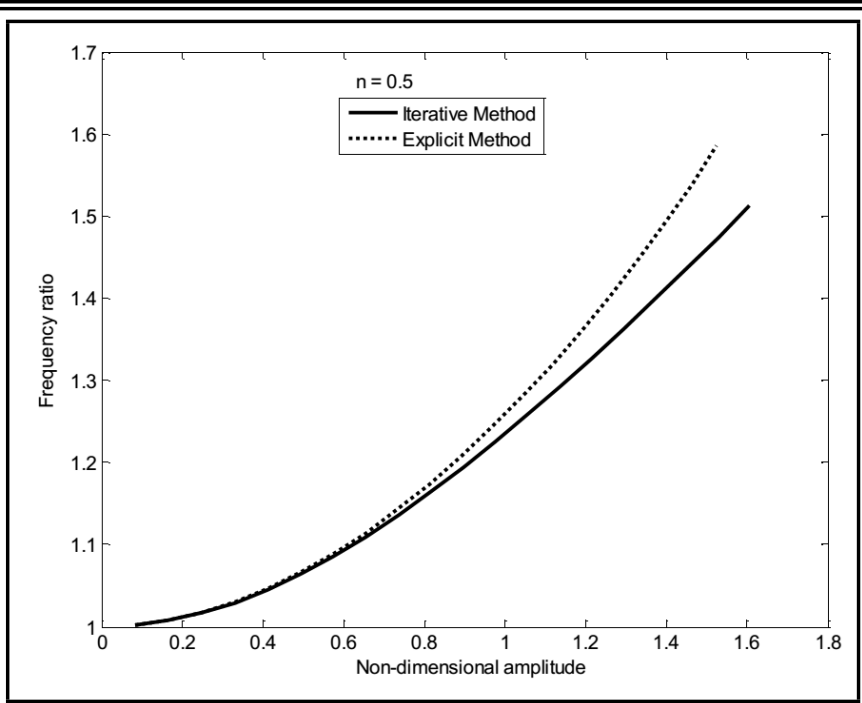

Figure 13. Comparison of frequencies parameter $\left(\omega_{n l}^{*} / \omega_{l}^{*}\right)$ for the first nonlinear clamped FG circular plate. (一): Iterative method of solution. (- - -): Explicit analytical solution.

Table 7. Comparison between values of frequency parameter $\left(\omega_{n l}^{*} / \omega_{l}^{*}\right)$ associated with the first non-linear axisymmetric mode shape of a clamped FG circular plate, obtained by iterative and explicit analytical solution at various values of the maximum non-dimensional amplitude $w_{\max }^{*}$.

\begin{tabular}{|c|c|c|c|}
\hline \hline \multicolumn{4}{|c|}{ Non-linear frequency ratio $\left(\omega_{n l}^{*} / \omega_{l}^{*}\right)$} \\
\hline$w_{\max }^{*}$ & Iterative solution & Explicit solution & Deviation (\%) \\
\hline 0.5 & 1.0662 & 1.0678 & 0.1500 \\
0.6 & 1.0939 & 1.0971 & 0.2925 \\
0.7 & 1.1252 & 1.1308 & 0.4976 \\
0.8 & 1.1603 & 1.1692 & 0.7670 \\
0.9 & 1.1984 & 1.2118 & 1.1181 \\
1.0 & 1.2393 & 1.2583 & 1.5331 \\
1.1 & 1.2831 & 1.3088 & 2.0029 \\
1.2 & 1.3291 & 1.3628 & 2.5355 \\
1.5 & 1.4610 & 1.5217 & 4.1546 \\
\hline
\end{tabular}

Eq. (55) into Eq. (54) leads to

$$
\varepsilon_{r}=\frac{3 a_{1}^{3} b_{111 r}^{*}}{2\left(k_{11}^{*}+\frac{3}{2} a_{1}^{2} b_{1111}^{*}-k_{r r}^{*}\right)}
$$

where $r=2, \ldots, 6$.

Equation (56) is an explicit simple formula, allowing direct calculation of the higher mode contributions to the first nonlinear mode shape of the FG circular plate as functions of the assigned first mode contribution $a_{1}$ and the known parameters $k_{r r}^{*}$ and $b_{111 r}^{*}$ (given in Table 6). Then, defines the first nonlinear amplitude-dependent FG circular plate mode shape $w_{n l 1}^{*}\left(r^{*}, a_{1}\right)$ is given as a series involving the circular plate modal parameters depending on the first six axisymmetric functions $w_{1}^{*}\left(r^{*}\right), w_{2}^{*}\left(r^{*}\right), \ldots, w_{6}^{*}\left(r^{*}\right)$ :

$$
\begin{aligned}
w_{n l 1}^{*}\left(r^{*}, a_{1}\right)= & a_{1} w_{1}^{*}\left(r^{*}\right)+ \\
& \sum_{r=2}^{6} \frac{3 a_{1}^{3} b_{111 r}^{*}}{2\left(k_{11}^{*}+\frac{3}{2} a_{1}^{2} b_{1111}^{*}-k_{r r}^{*}\right)} w_{r}^{*}\left(r^{*}\right)
\end{aligned}
$$

in which the predominant term, proportional to the first linear mode shape, is $a_{1} w_{1}^{*}\left(r^{*}\right)$, and the other terms, proportional to the higher linear mode shapes $\varepsilon_{2} w_{2}^{*}\left(r^{*}\right), \ldots, \varepsilon_{6} w_{6}^{*}\left(r^{*}\right)$, are the corrections due to the nonlinearity.

It may be seen in Figs. 11(a)-11(e) in which the higher mode contributions obtained by the explicit approximate solution are plotted against the maximum non-dimensional vibration am- 


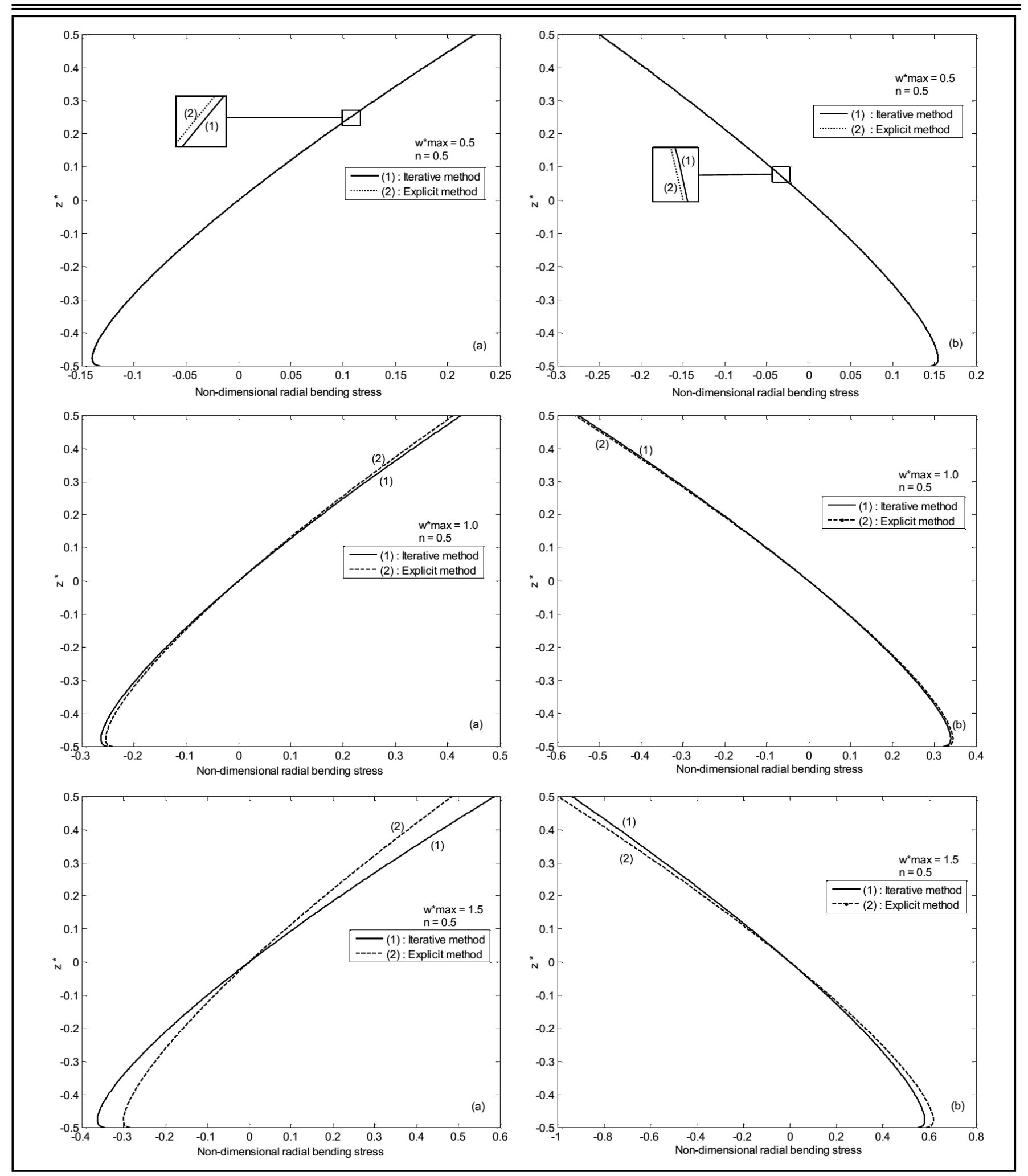

Figure 14. Comparison between dimensionless radial bending stresses distribution along the thickness, at the clamped Center (a) and the clamped Edge (b) of the FG circular plate for various non-dimensional vibration amplitudes. (-): Iterative method of solution. (- - -): Explicit analytical solution.

plitude and compared with that obtained by the iterative solution.

\subsubsection{Validity Domain of the Analytical Solutions}

The explicit analytical method of solution applied here to the first nonlinear mode shape of a clamped FG circular plate appears to be very appropriate for the analysis of geometrically nonlinear free vibration problems. Since it is based on an assumption concerning the order of magnitude of the basic function contribution coefficients, its domain of validity has to be delimited.

To have an accurate conclusion concerning this domain of validity, especially in engineering applications, a criterion was adopted based on the effect of the assumptions made on the mode shape, the nonlinear frequencies, and the radial bending stress obtained at the clamped edge and center of the FG circular plate are examined.

The normalized first nonlinear axisymmetric mode shape, 
obtained by numerical iterative solution and explicit analytical solution, is plotted in Figs. 12(a)-12(c) for various values of the maximum dimensionless vibration amplitude. It can be observed that the nonlinear effect increases with increasing the amplitude of vibration, and it may be seen from these figures that the first normalized nonlinear mode shape obtained by explicit approximate solution is in excellent agreement with that obtained by the iterative method of solution for maximum dimensionless vibration amplitude up to 1 and 0.5 , respectively.

In Fig. 13, the nonlinear frequency estimates, calculated using the single-function formula in Eq. (55) and the complete formula in Eq. (41), are plotted against the maximum dimensionless vibration amplitude, for the axisymmetric mode shape and the numerical results thus obtained for various values of the maximum non-dimensional amplitude are listed in Table 7. Table 7 and Fig. 13 show that the single-mode approach gives a good estimate of nonlinear frequency parameter $\left(\omega_{n l}^{*} / \omega_{l}^{*}\right)$ with a percentage error induced by the explicit approach solution that does not exceed $4.20 \%$ for the first axisymmetric mode shape at $w_{\max }^{*}=1.5$ compared with the one given by Eq. (41).

The dimensionless radial bending stress distribution along the thickness is associated with the first nonlinear axisymmetric mode shape obtained by the iterative method and that obtained by the analytical method and are plotted in Figs. 14(a) and 14(b) for various maximum dimensionless vibration amplitude. It may be seen from these figures that the radial bending stress obtained by the two approaches are in excellent agreement for maximum dimensionless vibration amplitude up to 1 and 0.5 for the first nonlinear axisymmetric FG circular plate mode shape. As may be seen in the corresponding figures, the error increases with increasing the vibration amplitude. For example, the error induced by the first formulation in the bending stress, corresponding to the first nonlinear mode, at the clamped edge of the FG circular plate, is 0.10 for a dimensionless amplitude of vibration equal to 0.5 times the plate thickness and does not exceed $6.0 \%$ for a dimensionless amplitude of vibration equal to 1.5 . It can then be concluded that the explicit analytical method gives acceptable results with respect to the nonlinear bending stress estimates for vibration amplitudes up to once the plate thickness for the first nonlinear mode shape.

\section{CONCLUSIONS}

The nonlinear free vibrations of FG circular plates have been examined using a theoretical model for geometrically nonlinear free vibrations. The model based on Hamilton's principle reduces the nonlinear free vibration problem to solution of a set of nonlinear algebraic equations. The amplitude dependence of the first nonlinear mode shape of clamped FG circular plates and the associated nonlinear parameters has been obtained via iterative solution of a set of nonlinear algebraic equations, involving a fourth order tensor due to the geometrical nonlinearity.

Considering the results obtained, numerical data corresponding to various values of the volume fraction index $n$ are plotted and discussed. Also, the results show that the nonlinear frequency increases with increasing vibration amplitudes, and all curves show the amplitude dependence of the stress distribution and a higher increase of the bending stress near to the clamps compared with the rate of increase obtained in the linear theory.

In order to obtain explicit analytical solutions for the first nonlinear axisymmetric mode shape of the FG circular plates, which are expected to be very useful in engineering applications and in further analytical developments, the improved version of the semi-analytical model developed by El Kadiri, Benamar, and White ${ }^{28}$ and El Kadiri and Benamar ${ }^{29}$ for beams and rectangular plates has been developed and adapted for the FG circular plate, which are shown to be in a good agreement with the iterative method.

Further investigations are needed to determine the membrane stress distribution at large vibration amplitudes by taking into account in the theory the effects of the in-plane displacements. It is also necessary to carry out a parametric study concerning the effect of the graded material properties such as Young's modulus $E$ and the mass density $\rho$ on the nonlinear vibration behavior of the plate.

\section{REFERENCES}

1 Koizumi, M. Functionally gradient materials the concept of FGM, Ceramic Transactions, 34, 3-10, (1993).

2 Allahverdizadeh, A., Naei, M. H., and Bahrami, M. N. Nonlinear free and forced vibration analysis of thin circular functionally graded plates, Journal of Sound and Vibration, 310 (4-5), 966-984, (2008). http://dx.doi.org/10.1016/j.jsv.2007.08.011

3 Praveen, G. N. and Reddy, J. N. Nonlinear transient thermo elastic analysis of functionally graded ceramic-metal plates, International Journal of Solids and Structures, 35 (33), 4457-4476, (1998). http://dx.doi.org/10.1016/s00207683(97)00253-9

4 Yang, J. and Shen, H.-S. Dynamic response of initially stressed functionally graded rectangular thin plates, Composite Structures, 54 (4), 497-508, (2001). http://dx.doi.org/10.1016/s0263-8223(01)00122-2

5 Yang, J. and Shen, H.-S. Vibration characteristics and transient response of shear-deformable functionally graded plates in thermal environments, Journal of Sound and Vibration, 255 (3), 579-602, (2001). http://dx.doi.org/10.1006/jsvi.2001.4161

6 Huang, X.-L. and Shen, H.-S. Nonlinear vibration and dynamic response of functionally graded plates in thermal environment, International Journal of Solids and Structures, 41 (9-10), 2403-2427, (2004). http://dx.doi.org/10.1016/j.ijsolstr.2003.11.012

7 Reddy, J. N. and Cheng, Z. Q. Frequency of functionally graded plates with three-dimensional asymptotic approach, Journal of Engineering Mechanics, 129 (8), 896-900,(2003). http://dx.doi.org/10.1061/(asce)07339399(2003)129:8(896)

8 Prakash, T. and Ganapathi, M. Asymmetric flexural vibration and thermoelastic stability of FGM circular plates using finite element method, Composites Part B: Engineering, 37 (7-8), 642-649, (2006). http://dx.doi.org/10.1016/j.compositesb.2006.03.005 
9 Efraim, E. and Eisenberger, M. Exact vibration analysis of variable thickness thick annular isotropic and FGM plates, Journal of Sound and Vibration, 299 (4-5), 720738, (2007). http://dx.doi.org/10.1016/j.jsv.2006.06.068

10 Dong, C. Y. Three-dimensional free vibration analysis of functionally graded annular plates using the ChebyshevRitz method, Materials and Design, 29 (8), 1518-1525, (2008). http://dx.doi.org/10.1016/j.matdes.2008.03.001

11 Malekzadeh, P., Atashi, M. M., and Karami, G. In-plane free vibration of functionally graded circular arches with temperature-dependent properties under thermal environment, Journal of Sound and Vibration, 326 (3-5), 837-851, (2009). http://dx.doi.org/10.1016/j.jsv.2009.05.016

12 Viswanathan, K. K., Javed, S., Aziz, Z. A., and Prabakar, $K$. Free vibration of symmetric angle-ply laminated annular circular plate of variable thickness under shear deformation theory, Meccanica, 50 (12), 3013-3027, (2015). http://dx.doi.org/10.1007/s11012-015-0175-3

13 Ebrahimi, F., Naei, M. H., and Rastgoo, A. Geometrically nonlinear vibration analysis of piezoelectrically actuated FGM plate with an initial large deformation, Journal of Mechanical Science and Technology, 23 (8), 2107-2117, (2009). http://dx.doi.org/10.1007/s12206-009-0358-8

14 Kermani, I. D., Ghayour, M., and Mirdamadi, H. R. Free vibration analysis of multi-directional functionally graded circular and annular plates, Journal of Mechanical Science and Technology, 26 (11), 3399-3410, (2012). http://dx.doi.org/10.1007/s12206-012-0860-2

15 Viswanathan, K. K., Javed, S., and Aziz, Z. A. Free vibration of antisymmetric angle-ply laminated annular circular plate, Lecture Notes in Engineering and Computer Science, 3 LNECS, 2136-2141, (2013).

16 Haterbouch, M. and Benamar, R. The effects of large vibration amplitudes on the axisymmetric mode shapes and natural frequencies of clamped thin isotropic circular plates, Part I: Iterative and explicit analytical solution for nonlinear transverse vibrations, Journal of Sound and Vibration, 265 (1), 123-154, (2003). http://dx.doi.org/10.1016/s0022$460 x(02) 01443-8$

17 Haterbouch, M. and Benamar, R. The effects of large vibration amplitudes on the axisymmetric mode shapes and natural frequencies of clamped thin isotropic circular plates, Part II: Iterative and explicit analytical solution for nonlinear coupled transverse and in-plane vibrations, Journal of Sound and Vibration, 277 (1-2), 1-30, (2004). http://dx.doi.org/10.1016/j.jsv.2003.08.039

18 Shen, H.-S. Functionally Graded Materials, CRC Press, (2009). http://dx.doi.org/10.1201/9781420092578

19 Timoshenko, S., Weinsowsky-Krieger, S., and Jones, R. M. Mechanics of Composite Materials, McGraw-Hill Kogakusha, Ltd. Tokyo, (1975), International Student Edition, Vol. 51.
20 Benamar, R., Bennouna, M. M. K., and White, R. G. The effects of large vibration amplitudes on the mode shapes and natural frequencies of thin elastic structures, Part II: Fully clamped rectangular isotropic plates, Journal of Sound and Vibration, 164 (2), 295-316, (1993). http://dx.doi.org/10.1006/jsvi.1993.1215

21 El Kadiri, M., Benamar, R., and White, R. G. The nonlinear free vibration of fully clamped rectangular plates: Second nonlinear mode for various plate aspect ratios, Journal of Sound and Vibration, 228 (2), 333-358, (1999). http://dx.doi.org/10.1006/jsvi.1999.2410

22 Benamar, R., Bennouna, M. M. K., and White, R. G. The effects of large vibration amplitudes on the mode shapes and natural frequencies of thin elastic structures, Part III: Fully clamped rectangular isotropic platesmeasurements of the mode shape amplitude dependence and the spatial distribution of harmonic distortion, Journal of Sound and Vibration, 175 (3), 377-395, (1994). http://dx.doi.org/10.1006/jsvi.1994.1335

23 El Bikri, K., Benamar, R., and Bennouna, M. Geometrically nonlinear free vibrations of clamped simply supported rectangular plates. Part I: The effects of large vibration amplitudes on the fundamental mode shape, Computers and Structures, 81 (20), 2029-2043, (2003). http://dx.doi.org/10.1016/s0045-7949(03)00152-4

24 Sridhar, S., Mook, D. T., and Nayfeh, A. H. Nonlinear resonances in the forced responses of plates, Part I: Symmetric responses of circular plates, Journal of Sound and Vibration, 41 (3), 359-373, (1975). http://dx.doi.org/10.1016/s0022-460x(75)80182-9

25 Leissa, A. W. Vibrations of Plates, NASA SP-160, U.S. Government Printing Office, Washington, DC, (1969).

26 Yamaki, N. Influence of large amplitudes on flexural vibrations of elastic plates, ZAMM - Journal of Applied Mathematics and Mechanics, 41 (12), 501-510, (1961). http://dx.doi.org/10.1002/zamm.19610411204

27 Nowinski, J. L. Nonlinear transverse vibrations of circular elastic plates built-in at the boundary, Proc. of the Fourth US National Congress on Applied Mechanics, 1, 325-334, (1962).

28 El Kadiri, M., Benamar, R., and White, R. G. Improvement of the semi-analytical method, for determining the geometrically nonlinear response of thin straight structures. Part I: application to clamped-clamped and simply supportedclamped beams, Journal of Sound and Vibration, 249 (2), 263-305, (2002). http://dx.doi.org/10.1006/jsvi.2001.3808

29 El Kadiri, M. and Benamar, R. Improvement of the semianalytical method, for determining the geometrically nonlinear response of thin straight structures. Part II: first and second nonlinear mode shapes of fully clamped rectangular plates, Journal of Sound and Vibration, 257 (1), 19-62, (2002). http://dx.doi.org/10.1006/jsvi.2002.5026 